\title{
Cylindrically symmetric scalar waves in general relativity
}

\author{
Shri Ram and S K Tiwari \\ Department of Applied Mathematics, Institute of Technology, \\ Banaras Hindu University, Varanası 221005 , India \\ Received I2 February 1998, accepted II March 1998
}

\begin{abstract}
In this note, exact solutions of Einstein equations with scalar waves are obtained for the most general cylindrically symmetric space-tıme which reduce to essentially statıc forms. The asymptotic behaviour of the null geodesic near the curvature singularity of a solution is discussed. The other solution is found to have no finite curvature singularity

Keywords : Einstein equations with scalar waves, cylindncally symmetric spuce-time, exact solutions
\end{abstract}

PACS No. : $\quad 04.20 . \mathrm{Jb}$

General relativity couples gravity with all fields. The study of the exact solutions of gravity coupled to other fields is important to understand clearly the physical and mathematical structures of space-times. For many reasons, the study of Einstein equations in the presence of scalar fields has been an object of special attention and various aspects of the problem have been investigated by Brahmchary [1], Bergmann and Leipnik [2], Buchdahl [3], Janis et al [4], Penny [5], Gautreau [6] and others. Most of the authors have taken up the problems of interacting gravitational and scalar fields with and without the rest-mass term. Several physically acceptable scalar-tensor theories of gravitation have been proposed and are widely studied by many workers. Scalar-tensor theories of gravitation provide the most natural generalisations of general relativity and thus provide a convenient set of representations for the observational limits on possible deviations from general relativity.

The most general spherically symmetric static solution of Einstein equations coupled with mass-less scalar field was found by Wyman [7]. Since then, some authors investigated 
its global properties and a few interesting results were found. Roberts [8] has discussed the applications of spherically symmetric solutions of the mass-less scalar Einstein equations to cosmic censorship and has given a non-static solution to the field equations. He has also constructed the Vaidya form of Wyman solution obeying the reasonable energy conditions. Li and Liang [9] have presented the static general solution with plane symmetric scalar fields and have shown that the singularity in the plane symmetric case is not influenced essentially by the introduction of the scalar field. $\mathrm{Li}$ [10] has presented the general plane symmetric metric yielded by a scalar wave and concluded that the metric is either static or spatially homogeneous. He has shown that the Taub Theorem [11] can be generalised to space-time with a scalar wave. Shri Ram and Singh [12] have derived an exact non-static scalar wave solution for the cylindrically symmetric Marder [13] metric which give Taub solution [11] and $\mathrm{Li}$ solution [10] in special cases.

In this note, we consider Einstein equations with scalar wave for the most general cylindrically symmetric metric recently discussed by Banerjee et al [14] in the investigation of exact gravitational fields due to static and nonstatic cosmic strings arising due to the breaking of a global $U$ (1) symmetry. The field equations are completely integrated and two exact solutions are then presented which reduce tu essentially static form under coordinate transformations. We also discuss the asymptotic behaviour of the null geodesic near the singularity of one of the solutions. The other solution has no finite singularity.

\section{Field equations :}

The general cylindrically symmetric line element can be written as

$$
d s^{2}=e^{2(K-U)}\left(-d t^{2}+d r^{2}\right)+e^{2 U} d z^{2}+W^{2} e^{-2 U} d \theta^{2} .
$$

where all of $K, U$ and $W$ are functions of $r$ and $t$ [14]. Setting $x^{1}=r, x^{2}=z, x^{3}=\theta$ and $x^{4}=t$, the non-vanishing components of the Ricci tensor are $R_{11}, R_{22}, R_{33}, R_{44}$ and $R_{14}$.

The energy-momentum tensor for a massless scalar field is

$$
T_{\alpha \beta}=\phi_{, \alpha} \phi_{, \beta}-\frac{1}{2} g_{\alpha \beta} \phi_{, \gamma} \phi^{\prime} \gamma,
$$

where the scalar field $\phi$ is the solution of Klein-Gordon equation :

$$
g^{\alpha \beta} \phi_{, \alpha \beta}=0 \text {. }
$$

A comma and a semicolon denote ordinary and covariant derivative respectively.

The Einstein equations are

$$
R_{\alpha \beta}-\frac{1}{2} g_{\alpha \beta} R=8 \pi T_{\alpha \beta} .
$$

On contraction, the field equations (4) can be written in the form

$$
R_{\alpha \beta}=8 \pi \phi, \alpha \phi_{\beta} .
$$


Because of the symmetry in the metric (1), $\phi$ is function of $r$ and $t$. For the line-element (1), the Einstein equations (5) give the following set of equations :

$$
\begin{aligned}
& -\frac{W_{11}}{W}-K_{11}+K_{44}+U_{11}-U_{44}+\frac{U_{1} W_{1}}{W}+\frac{K_{1} W_{1}}{W}-\frac{U_{4} W_{4}}{W} \\
& \quad+\frac{K_{4} W_{4}}{W}-2 U_{1}^{2}=8 \pi \phi_{1}^{2}, \\
& -\frac{W_{11}}{W}+\frac{W_{44}}{W}+U_{11}-U_{44}+\frac{U_{1} W_{1}}{W}-\frac{U_{4} W_{4}}{W}=0, \\
& -U_{11}+U_{44}-\frac{U_{1} W_{1}}{W}+\frac{U_{4} W_{4}}{W}=0, \\
& -\frac{W_{44}}{W}+K_{11}-K_{44}-U_{11}-U_{44}+\frac{K_{1} W_{1}}{W}-\frac{U_{1} W_{1}}{W}+\frac{K_{4} W_{4}}{W} \\
& \quad+\frac{U_{4} W_{4}}{W}-2 U_{4}^{2}=8 \pi \phi_{4}^{2}, \\
& -\frac{W_{14}}{W}+\frac{W_{1} W_{4}}{W}+\frac{K_{1} W_{4}}{W}-2 U_{1} U_{4}=8 \pi \phi_{1} \phi_{4} .
\end{aligned}
$$

The Klein-Gordon equation (3) leads to

$$
\phi_{11}-\phi_{44}+\frac{W_{1} \phi_{1}}{W}-\frac{W_{4} \phi_{4}}{W}=0
$$

A linear combination of eqs $(6-10)$ yields

$$
\begin{aligned}
& W_{44}-W_{11}=0 \text {, } \\
& -U_{11}+U_{4}-\frac{U_{1} w_{1}}{W}+\frac{U_{4} W_{4}}{W}=0, \\
& -\frac{W_{11}}{W}-\frac{W_{44}}{W}+\frac{2 K_{1} W_{1}}{W}+\frac{2 K_{4} W_{4}}{W}-2 U_{1}^{2}-2 U_{4}^{2} \\
& =8 \pi\left[\phi_{1}^{2}+\phi_{4}^{2}\right] \text {. } \\
& K_{44}-K_{11}+U_{11}-U_{44}-U_{1}^{2}+U_{4}^{2}+\frac{U_{1} W_{1}}{W}-\frac{U_{4} W_{4}}{W} \\
& =4 \pi\left[\phi_{1}^{2}-\phi_{4}^{2}\right] \text {. } \\
& \text { and } \quad-\frac{W_{14}}{W}+\frac{W_{1} K_{4}}{W}+\frac{W_{4} K_{1}}{W}-2 U_{1} U_{4}=8 \pi \phi_{1} \phi_{4} \text {. }
\end{aligned}
$$

Herc suffixes 1 and 4 are differentiation with respect to $r$ and $t$ respectively.

Solutions of the field equations :

The general solution of $(12)$ is

$$
W=w_{1} \dot{(\xi)}+w_{2}(\eta), \quad \text { where } \xi=t+r, \eta=t-r .
$$


Let the scalar field $\phi$ be the solution of wave equation, which is referred to as a scalar wave.

$$
\phi=h_{1}(\xi)+h_{2}(\eta)
$$

Using (17) and (18) in (11) we obtain

$$
\underset{d \xi}{d h_{1}} / \frac{d w_{1}}{d \xi}=-\frac{d h_{2}}{d \eta} / \begin{gathered}
d w_{2} \\
d \eta
\end{gathered}=a,
$$

where $a$ is an arbitrary constant. In view of these equations, the scalar field $\phi$ becomes

$$
\phi=a\left\{w_{1}(\xi)-w_{2}(\eta)\right\}+b,
$$

$b$ being another arbitrary constant. From eqs. (12) and (13), we can write

$$
U=\epsilon \log W, \quad \epsilon= \pm 1
$$

Using (17), (18) and (19) in eq. (15), we obtain

$$
\frac{\partial^{2} K}{\partial \xi \partial \eta}=4 \pi a^{2} w_{1}^{\prime}(\xi) w_{2}^{\prime}(\eta)-\frac{w_{1}^{\prime}(\xi) w_{2}^{\prime}(\eta)}{\left[w_{1}(\xi)+w_{2}(\eta)\right]^{2}} .
$$

A dash denotes ordinary derivative. Eq. (21) has the general solution

$$
K=4 \pi a^{2} w_{1}(\xi) w_{2}(\eta)+\log \left\{w_{1}(\xi)+w_{2}(\eta)\right\}+g_{1}(\xi)+g_{2}(\eta),
$$

where $g_{1}(\xi)$ and $g_{2}(\eta)$ are arbitrary functions. Substituting $K$ from (22) into (14) and (16), we obtain

$$
\begin{aligned}
w_{1}^{\prime}(\xi) g_{1}^{\prime}(\xi) & +w_{2}^{\prime}(\eta) g_{2}^{\prime}(\eta)=\frac{1}{2}\left[w_{1}^{\prime \prime}(\xi)+w_{2}^{\prime \prime}(\eta)\right] \\
& +4 \pi a^{2}\left[\left\{w_{1}^{\prime}(\xi)\right\}^{2} w_{1}(\xi)+\left\{w_{2}^{\prime}(\eta)\right\}^{2} w_{2}(\eta)\right] \\
w_{1}^{\prime}(\xi) g_{1}^{\prime}(\xi) & -w_{2}^{\prime}(\eta) g_{2}^{\prime}(\eta)=\frac{1}{2}\left[w_{1}^{\prime \prime}(\xi)-w_{2}^{\prime \prime}(\eta)\right] \\
& +4 \pi a^{2}\left[\left\{w_{1}^{\prime}(\xi)\right\}^{2} w_{1}(\xi)-\left\{w_{2}^{\prime}(\eta)\right\}^{2} w_{2}(\eta)\right]
\end{aligned}
$$

which are equivalent to

$$
\begin{array}{ll} 
& g_{1}(\xi)=\frac{1}{2} \log w_{1}^{\prime}(\xi)+2 \pi a^{2}\left\{w_{1}(\xi)\right\}^{2}+\frac{1}{2} \log c_{1} \\
\text { and } \quad & g_{2}(\eta)=\frac{1}{2} \log w_{2}^{\prime}(\eta)-2 \pi a^{2}\left\{w_{2}(\eta)\right\}^{2}+\frac{1}{2} \log c_{2} .
\end{array}
$$

Equations (25) and (26) can be written as

$$
\begin{aligned}
& e^{2 g_{1}}=c_{1}\left|w_{1}^{\prime}\right| e^{4 \pi u^{2} w_{1}^{2}}= \pm c_{1} w_{1}^{\prime} e^{4 \pi a^{2} w_{1}^{2}}, \\
& e^{2 k_{2}}=c_{2}\left|w_{2}^{\prime}\right| e^{4 \sigma^{2} w_{2}^{2}}= \pm c_{2} w_{2}^{\prime} e^{4 w^{2} w_{2}^{2}} .
\end{aligned}
$$


Case I: when $\epsilon=-1$

From eqs. (20), (22), (27) and (28), we obtain

$$
e^{2 K-2 U}= \pm c^{2}\left[w_{1}(\xi)+w_{2}(\eta)\right]^{4} w_{1}^{\prime}(\xi) w_{2}^{\prime}(\eta) e^{4 x n^{2}\left(w_{1}(\xi)+w_{2}(\eta)\right]^{2}} .
$$

where we take the negative sign if $w_{1}^{\prime} w_{2}^{\prime}<0$ and $c^{2}=c_{1} c_{2}$ is a constant.

The metric of the solution becomes

$$
\begin{aligned}
d s^{2}= & \pm c^{2}\left[w_{1}(\xi)+w_{2}(\eta)\right]^{4} w_{1}^{\prime}(\xi) w_{2}^{\prime}(\eta) e^{\left.4 \pi u^{2} \mid w_{1}(\xi)+w_{2}(\eta)\right]^{2}(d \xi d \eta)} \\
& +\left[w_{1}(\xi)+w_{2}(\eta)\right]^{-2} d z^{2}+\left[w_{1}(\xi)+w_{2}(\eta)\right]^{4} d \theta^{2}
\end{aligned}
$$

Using scale transformation,

$$
w_{1}(\xi)=R+T \text { and } w_{2}(\eta)=R-T \text {. }
$$

the cylindrically symmetric line element (1) yielded by a scalar wave can be written in the lorm

$$
d s^{2}=c^{2} R^{4} e^{4 \pi a^{2} R^{2}}\left(d R^{2}-d T^{2}\right)+R^{-2} d z^{2}+R^{4} d \theta^{2} .
$$

The scalar curvature of space-time (31) has the value $32 \pi a^{2} / R^{4} e^{4 \pi a^{2} R^{2}}$ which tends to infinity as $R \rightarrow 0$. Thus $R=0$ is a scalar curvature singularity. Investigating the asymptotic hehaviour of the null geodesic, it is found that the null geodesics approaching $R=0$ in $T-R$ plane are incomplete.

Case II: when $\epsilon=+1$

From eqs. (20), (22), (27) and (28), we obtain

$$
e^{2 K-2 U}= \pm c^{2} w_{1}^{\prime}(\xi) w_{2}^{\prime}(\eta) e^{4 \pi a^{2}\left[w_{1}(\xi)+w_{2}(\eta)\right]^{2}},
$$

where we take the negative sign if $w_{1}^{\prime} w_{2}^{\prime}<0$ and $c^{2}=c_{1} c_{2}$ is a constant.

The metric of the solution becomes

$$
\begin{aligned}
d s^{2} & =c^{2} w_{1}^{\prime}(\xi) w_{2}^{\prime}(\eta) e^{4 \pi d^{2}\left(w_{1}(\xi)+w_{2}(\eta)\right]^{2}} d \xi d \eta \\
& +\left[w_{1}(\xi)+w_{2}(\eta)\right]^{2} d z^{2}+d \theta^{2} .
\end{aligned}
$$

Using scale transformation,

$$
w_{1}(\xi)=R+T \text { and } w_{2}(\eta)=R-T,
$$

the cylindrically symmetric line element (1) yielded by a scalar wave can be written as

$$
d s^{2}=c^{2} e^{4 \pi u^{2} R^{2}}\left(d R^{2}-d T^{2}\right)+R^{2} d z^{2}+d \theta^{2} .
$$

The scalar curvature of space-time (34) is $32 \pi a^{2} / e^{4 \pi u^{2} R^{2}}$ which shows that the metric (34) has no finite singularity.

\section{Kcferences}

(1) RL Brahmchary Prog. Theor. Phys. 23749 (1960)

12] O Bergmann and R Leipnik Phys Rev. 107 I157 (1957) 
[3] H A Buchdahl Phys. Rev. 115 I32.5 (1959)

[4] A Janis, E T Newman and J Winicour Phys. Rev Lett. 20878 (1968)

[5] R Penny Phys Rev. 1741578 (1968)

16] R Gautreau Nuovo Cim B62 360 (1969)

[7] M Wyman Phys. Rev. D24 839 (1981)

[8] M D Roberts Gen. Rel Grav 17913 (1985)

19] Jaan-Zeng Ll and Can-bin Liang Acra Phys. Sinica 40643 (1991)

[10] Jian-Zeng Lı J. Marh. Phys. 333506 (1992)

(1I) A H Taub Ann. Math 53472 (1951)

[12] Shri Ram and J K Singh IL. Nuovo Cim. BIII 7.57 (1996)

[I 1] L Marder Proc. Roy Soc. A244 524 (1958)

[14] A Banerjee, N Banerjee and A A Sen Phys. Rev D53 5.508 (1996) 